\title{
Transcriptional Response and Enhanced Intestinal Adhesion Ability of Lactobacillus rhamnosus GG after Acid Stress ${ }^{\text {[ }}$
}

\author{
Miseon Bang ${ }^{1 \dagger}$, Cheng-Chung Yong ${ }^{1 \dagger}$, Hyeok-Jin $\mathrm{Ko}^{2,3}$, In-Geol Choi ${ }^{2}$, and Sejong $\mathrm{Oh}^{1 *}$ \\ ${ }^{1}$ Division of Animal Science, Chonnam National University, Gwangju 61186, Republic of Korea \\ ${ }^{2}$ Department of Biotechnology, College of Life Sciences and Biotechnology, Korea University, Seoul 02841, Republic of Korea \\ ${ }^{3}$ Food RED Center, Samyang Corp., Seongnam 13488, Republic of Korea
}

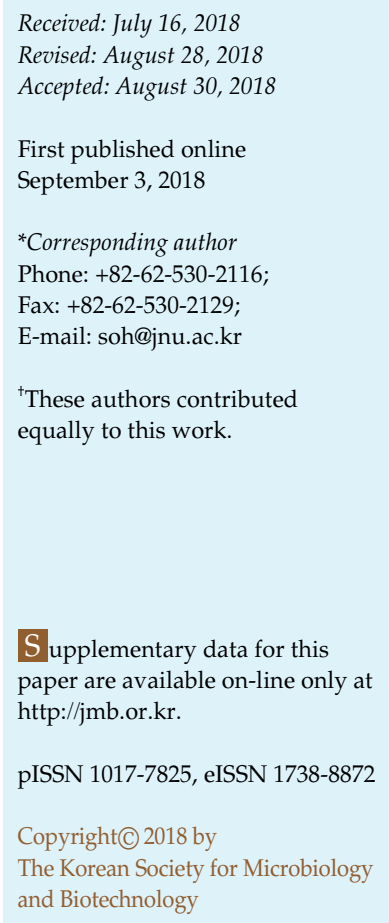

Lactobacillus rhamnosus GG (LGG) is a probiotic commonly used in fermented dairy products. In this study, RNA-sequencing was performed to unravel the effects of acid stress on LGG. The transcriptomic data revealed that the exposure of LGG to acid at $\mathrm{pH} 4.5$ (resembling the final $\mathrm{pH}$ of fermented dairy products) for $1 \mathrm{~h}$ or $24 \mathrm{~h}$ provoked a stringent-type transcriptomic response wherein stress response- and glycolysis-related genes were upregulated, whereas genes involved in gluconeogenesis, amino acid metabolism, and nucleotide metabolism were suppressed. Notably, the pilus-specific adhesion genes, spaC, and spaF were significantly upregulated upon exposure to acid-stress. The transcriptomic results were further confirmed via quantitative polymerase chain reaction analysis. Moreover, acid-stressed LGG demonstrated an enhanced mucin-binding ability in vitro, with $1 \log$ more LGG cells $(p<0.05)$ bound to a mucin layer in a 96-well culture plate as compared to the control. The enhanced intestinal binding ability of acid-stressed LGG was confirmed in an animal study, wherein significantly more viable LGG cells ( $\geq 2 \log$ CFU/g) were observed in the ileum, caecum, and colon of acid-stressed LGG-treated mice as compared with a non-acid-stressed LGG-treated control group. To our knowledge, this is the first report showing that acid stress enhanced the intestine-binding ability of LGG through the induction of pili-related genes.

Keywords: Stress response, Lactobacillus, transcriptomics, probiotics, microbial physiology, adhesion

\section{Introduction}

Fermented dairy products have been consumed for centuries, and yogurt is one of the most popular fermented milk products. Generally, the final $\mathrm{pH}$ of fermented dairy products is relatively low (approximately $\mathrm{pH} 4.5$ ) owing to the accumulation of fermentation end products, especially lactic acid. This low-pH environment might affect the survival of lactic acid bacteria within fermented milk. The ability to withstand an acidic environment is crucial for the survival and commercial utilization of probiotic bacteria. For instance, a previous study reported that certain Lactobacillus strains could survive up to 52 weeks in a low$\mathrm{pH}$ environment such as that in commercially available yogurts with only a slight reduction in cell viability [1].
During fermentation, the lactic acid produced by Lactobacillus strains accumulates and acidifies the environment; subsequently, the undissociated form of lactic acid reenters the cytoplasm via simple diffusion [2]. Upon entering the cytoplasm, lactic acid dissociates into protons and anions, disrupts the intracellular $\mathrm{pH}$ and anion pool, and impairs normal cellular function once its concentration exceeds the cytoplasmic buffering capacity [3-5]. Several mechanisms are employed by bacteria to counteract the acid challenge, including the proton-translocating $\mathrm{F}_{1} \mathrm{~F}_{0^{-}}$ ATPase that constantly pumps protons out of the bacterial cytoplasm to maintain the neutral $\mathrm{pH}$ of the bacterial cytosol [6-8]. Furthermore, several stringent responses occur in bacteria during acid stress, including the activation of genes involved in amino acid biosynthesis and transport 
to counteract amino acid starvation [9]. For instance, the $\operatorname{cplCEL}$ chaperones degrade or repair stress-damaged proteins, and biosynthesize peptidoglycan and cell membrane components during acid stress [10-13].

After surviving acid stress in fermented dairy products and the gastrointestinal tract, it is essential for probiotic strains to adhere to the host mucosal surface, where they interact with the gut-associated lymphoid tissue and mediate local and systemic immune effects. As suggested by previous studies, only adherent probiotics can effectively induce immuno-modulatory effects and stabilize the host's gut mucosal barrier [14-16]. Lactobacillus rhamnosus strain GG (LGG) is a human intestinal isolate that has wellcharacterized probiotic benefits. Previous genomic analyses of LGG have revealed two pilus gene clusters (spaCBA and spaFED) that encode pili, which are proteinaceous cell surface-localized polymeric structures on LGG that mediate its binding to the human intestinal mucosa. LGG is the only known probiotic strain that possesses mucus-binding pili, and these structures provide LGG with competitive advantage in exerting its probiotic actions [17, 18]. Each pilus of LGG consists of major pilins (SpaA and SpaD) forming the pilus shaft, ancillary minor pilin subunits (SpaB and SpaE) decorating the pilus backbone, and large-sized minor pilin subunits (SpaC and SpaF) that typically serve as adhesive components located at the pilus tips [18-20].

In the present study, to decipher the metabolic responses in LGG under acid stress at $\mathrm{pH} 4.5$, which is similar to the $\mathrm{pH}$ of most fermented milk-based food products, the genetic responses of LGG were examined using RNAsequencing. Additionally, the adherent ability of LGG under acid stress was investigated via in vitro and in vivo approaches. Our findings provide important insights into the role of the pilus fiber of LGG in mediating adhesion to the intestinal mucosa during acid stress.

\section{Materials and Methods}

\section{Bacteria Cultivation and Cell Harvesting}

Lactobacillus rhamnosus strain GG ATCC 53103 was obtained from Valio, Helsinki, Finland, and maintained in de-Man, Rogosa and Sharpe (MRS) medium to mid-log phase (optical density at $600 \mathrm{~nm}\left[\mathrm{OD}_{600 \mathrm{~nm}}\right]$ of 0.6 , equivalent to approximately $3 \times 10^{8} \mathrm{CFU} / \mathrm{ml}$ ). Cell pellets were collected via centrifugation at 5,000 $\times g$ for $10 \mathrm{~min}$ at room temperature (Micro17TR, Hanil Scientific Co., Korea). The cell pellets were re-suspended in MRS broth at $\mathrm{pH} 4.5$ and incubated at $37^{\circ} \mathrm{C}$ for 1 or $24 \mathrm{~h}$. The control consisted of a cell pellet re-suspended in MRS broth at $\mathrm{pH} 7.0$ and incubated at $37^{\circ} \mathrm{C}$ for $1 \mathrm{~h}$. After incubation, the cultures were centrifuged and the cell pellets were collected. The collected cell pellets were stored at $-80^{\circ} \mathrm{C}$ until further use.

\section{RNA Sequencing and Data Analyses}

Total RNA was isolated from the collected cell pellets using RNeasy Mini Kits (Qiagen, USA), digested with RNase-freeDNase (Qiagen) to remove DNA, and depleted of ribosomal RNA using a Ribo-Zero rRNA Removal Kit (Illumina, USA) according to the manufacturer's instructions. Ten micrograms of extracted RNA was used to prepare a sequencing library using a TruSeq Stranded mRNA Library Prep Kit (Illumina) according to the manufacturer's instructions. RNA sequencing was performed with an Illumina MiSeq platform. All data sets have been deposited in the Gene Expression Omnibus database under accession number GSE107337. Data analysis was performed using EdgeR, Bioconductor components in R packages with LGG as the reference genome.

Validating the Overexpression of Adhesion-Related Genes in Acid-Stressed LGG by Quantitative Reverse-Transcription PCR

Total RNA was extracted from the collected cell pellets using RNeasy Mini Kits (Qiagen) and DNA was removed with RNasefree-DNase (Qiagen) according to the manufacturer's instructions. cDNA was synthesized from $1 \mu \mathrm{g}$ of the extracted RNA with an RT Premix Oligo (dt) Kit (Intron, Korea). The primers used in this study were designed using Primer3plus software (http://www. bioinformatics.nl/cgi-bin/primer3plus/primer3plus.cgi), based on the genome sequence of LGG (Table 1). Glyceraldehyde-3phosphate dehydrogenase $(G A P D H)$ was used as an internal control gene. Quantitative PCR (qPCR) was performed using KAPA SYBR FAST qPCR kit (KapaBiosystems, USA) in a thermal cycler (Bio-Rad Laboratories, USA) with the conditions of initial denaturation at $95^{\circ} \mathrm{C}$ for $5 \mathrm{~min}$, followed by 35 cycles of denaturation at $95^{\circ} \mathrm{C}$ for $10 \mathrm{sec}$, annealing at $54^{\circ} \mathrm{C}$ for $15 \mathrm{sec}$ and extension at $72^{\circ} \mathrm{C}$ for $15 \mathrm{sec}$. The expression of each gene was determined via a comparative analysis based on $\mathrm{Ct}$ values as follows:

Relative expression $=2^{-\Delta C \mathrm{Ct}}$ with $\mathrm{C}_{\mathrm{t}}=\mathrm{C}_{\mathrm{t} \text { gene }}-\mathrm{C}_{\mathrm{tGAPDH}}$.

\section{Mucin-Binding Property of Acid-Stressed LGG}

A 96-well microtiter plate was inoculated with $100 \mu \mathrm{l} \mathrm{of} 10 \mathrm{mg} / \mathrm{ml}$ mucin solution (Sigma-Aldrich, USA) and kept overnight at $4{ }^{\circ} \mathrm{C}$. Wells were then washed twice with $0.85 \% \mathrm{NaCl}$ prior to inoculation with LGG. LGG was grown in MRS broth until it reached an $\mathrm{OD}_{600 \mathrm{~nm}}$ of 0.6 and centrifuged to collect cell pellets. The cell pellets were re-suspended in acidic MRS broth ( $\mathrm{pH} 4.5)$ for 1 or $24 \mathrm{~h}$, or neutral MRS broth ( $\mathrm{pH} 7.0$; control) for $1 \mathrm{~h}$. $100 \mu \mathrm{l}$ of each culture $\left(10^{8} \mathrm{CFU} / \mathrm{ml}\right)$ was added to the microtiter wells and incubated for $2 \mathrm{~h}$ at $37^{\circ} \mathrm{C}$. Thereafter, the wells were washed five times with $0.85 \% \mathrm{NaCl}$ to remove unbound bacteria. Adherent bacteria were removed with $0.1 \%$ Triton X-100 solution, followed by serial 10-fold dilutions and then plated on MRS agar. Colonies were counted after incubation at $37^{\circ} \mathrm{C}$ for $48 \mathrm{~h}$. 
Table 1. Oligonucleotide primers used for the qRT-PCR analysis of adhesion-related gene expression.

\begin{tabular}{|c|c|c|c|c|}
\hline Gene & Sequence of PCR primers $\left(5^{\prime}\right.$ to $\left.3^{\prime}\right)$ & Target locus & Product & Reference \\
\hline spaF & $\begin{array}{l}\text { Forward : GCTGATGTTACTGCTGATGC } \\
\text { Reverse : ATCCGTATTTTGAACGGGTA }\end{array}$ & LRHM_2281 & putative cell surface protein & PMID 19820099 \\
\hline $\operatorname{spaC}$ & $\begin{array}{l}\text { Forward : CAACTTGATGGGACAACGTA } \\
\text { Reverse : TCTGGTGCTTTTGTTTCTGA }\end{array}$ & LRHM_0428 & Cell surface protein & PMID 19820099 \\
\hline pili & $\begin{array}{l}\text { Forward : GATTATCGGGTTGATTCTGG } \\
\text { Reverse : AAATCGCCTTCGTACATCTC }\end{array}$ & LGG_02339 & $\begin{array}{l}\text { Pili_PIN/TRAM domain-containing } \\
\text { protein }\end{array}$ & PMID 19820099 \\
\hline Pilus444 & $\begin{array}{l}\text { Forward : CAACTTGATGGGACAACGTA } \\
\text { Reverse : TTTGCAGGATTGCTTTGATA }\end{array}$ & LGG_00444 & Pilus protein & PMID 19820099 \\
\hline Pilus443 & $\begin{array}{l}\text { Forward : CTAAATCCTTCCGTCCGTTA } \\
\text { Reverse : CTCAACGTCGTTTGTGCTAC }\end{array}$ & LGG_00443 & Pilus protein & PMID 19820099 \\
\hline Gene & Sequence of PCR primers ( $5^{\prime}$ to $\left.3^{\prime}\right)$ & Target locus & Product & Reference \\
\hline Pilus442 & $\begin{array}{l}\text { Forward : GATGGTTCTCGGGTTTAATG } \\
\text { Reverse : ACCCACGTCAATCGATAGTT }\end{array}$ & LGG_00442 & pilus protein & PMID 19820099 \\
\hline Pilus2370 & $\begin{array}{l}\text { Forward : AACTATCCATTCGGGTTTGA } \\
\text { Reverse : GTTGTCGGATCAAGGATTTC }\end{array}$ & LGG_02370 & Pilus protein & PMID 19820099 \\
\hline$m b f$ & $\begin{array}{l}\text { Forward : TGAAGTTGGAAAAGCGTACA } \\
\text { Reverse : AGGAAAAGTTCCTGATGCTG }\end{array}$ & AB968049 & Mucus binding factor & PMID 19820099 \\
\hline GAPDH & $\begin{array}{l}\text { Forward : GATCGTTTCTGCAGGTTCTT } \\
\text { Reverse : CCGTTCAATTCTGGGATAAC }\end{array}$ & LGG_00933 & $\begin{array}{l}\text { Type I glyceraldehyde-3-phosphate } \\
\text { dehydrogenase }\end{array}$ & PMID 19820099 \\
\hline
\end{tabular}

In Vivo Assessment of the Intestine-Binding Ability of AcidStressed LGG

LGG cultures were grown in MRS broth until reaching an $\mathrm{OD}_{600 \mathrm{~nm}}$ of 0.6 and centrifuged to collect cell pellets. The cell pellets were re-suspended in acidic MRS broth ( $\mathrm{pH} 4.5)$ for $24 \mathrm{~h}$ or neutral MRS broth ( $\mathrm{pH} 7.0$; control) for $1 \mathrm{~h}$. The control consisted of an LGG culture grown overnight without acidic or neutral MRS broth treatment. Then, cell pellets were collected via centrifugation and incubated with fluorescein isothiocyanate (FITC) solution $(1 \mathrm{mg} / \mathrm{ml}$ in $50 \mathrm{mmol} / 1$ sodium carbonate buffer, $\mathrm{pH} 8.9)$ for $2 \mathrm{~h}$ at room temperature. The labeled cells were washed 5 times with $0.85 \% \mathrm{NaCl}$ to remove the remaining FITC and then orally administered to male C57BL/6J mice. After $12 \mathrm{~h}$, the mice were sacrificed, and their intestines were collected to detect the presence of adherent labeled cells using a confocal laser scanning microscope (FV500, Olympus, Japan). Animal experiments were conducted in accordance with the guidelines of the Institutional Animal Care and Use Committee at Chonnam National University (project number: CNUIACUC-YB-2012-40).

The number of viable LGG adhered to the intestines was also determined. Fifty milligram portions of ileum, cecum, and colon were mixed with an equal volume of phosphate buffer saline and homogenized using a tissue grinder (Thomas Scientific, USA). Serial 10-fold dilutions were made and the number of viable LGG was counted on glucose blood liver (BL) agar supplemented with vancomycin after being incubated for $48 \mathrm{~h}$ at $37^{\circ} \mathrm{C}$ [21].

\section{Statistical Analyses}

All values were expressed as the mean \pm standard deviation from triplicate runs $(n=3)$. Duncan's multiple range test was used to determine the significance of differences between means at a significance threshold of $p<0.05$.

\section{Results}

\section{Transcriptional Response to LGG to Acid Stress}

The effects of short-term $(1 \mathrm{~h})$ and long-term $(24 \mathrm{~h})$ acid stress condition $(\mathrm{pH} 4.5)$ on the global gene expression of LGG were investigated via RNA sequencing. Through normalization, analysis, and comparison of the global gene expression profiles of the acid-stressed LGG ( $\mathrm{pH} 4.5$ for 1 or $24 \mathrm{~h}$ ) and the control LGG (pH 7.0 for $1 \mathrm{~h}$ ), we identified 228 genes that were significantly $(p<0.05)$ altered in response to acid stress condition (Fig. 1A). Among the 228 genes affected by acid stress, 128 genes were significantly $(p<0.05)$ upregulated (Table S1) whereas 100 genes were significantly $(p<0.05)$ downregulated (Table S2).

Among the upregulated genes, 47\% (61/129) encoded for hypothetical proteins with unknown functions. Meanwhile, genes encoding phage-related proteins demonstrated the highest fold change upon acid stress exposure, followed by hypothetical proteins and DNA-related proteins (Fig. 1B). Based on the transcriptomic data, the stress responserelated genes LGG_02240 and LGG_01072, which encode the co-chaperonin GroES, and the CspA family cold shock protein, respectively, were detected and induced in response 
A

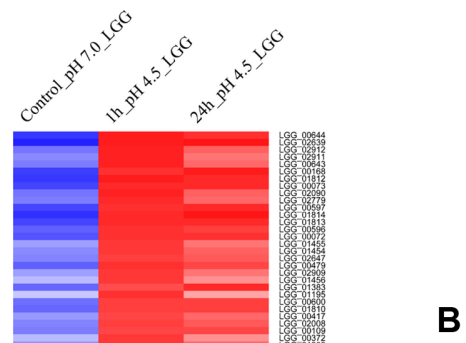

B

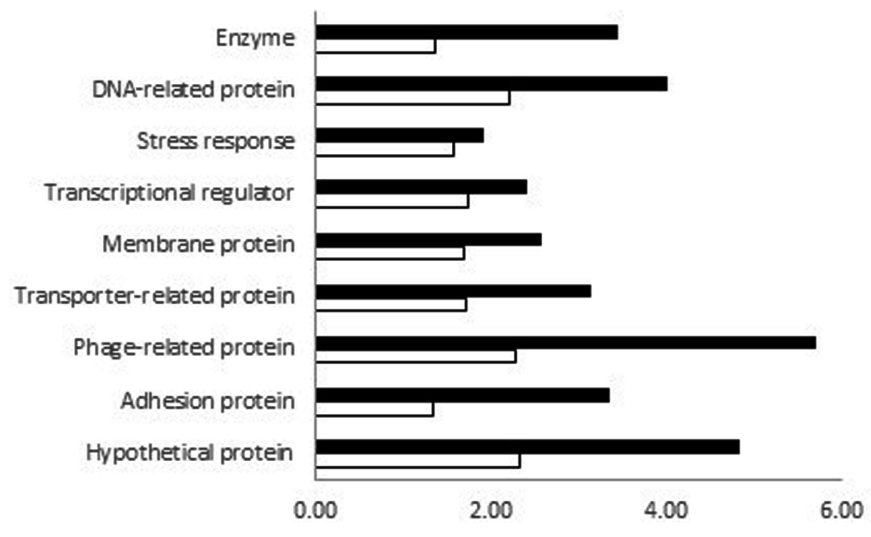

- pH $4.5 \_24 \mathrm{~h}$ apH $4.5 \_1 \mathrm{~h}$

C

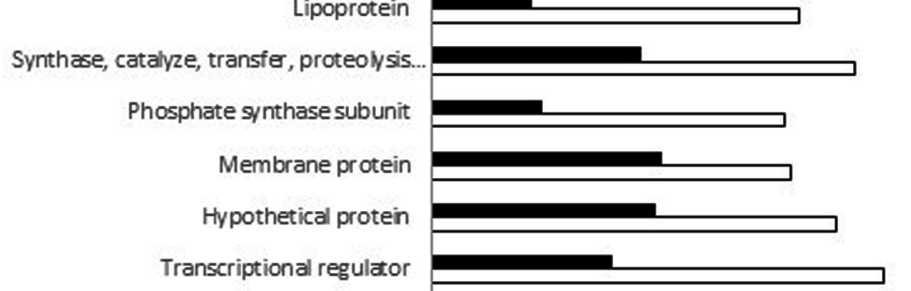

Nucleotide transport and metabolism

Amino acid transport, metabo lism

Carbohydrate transport, metabolism:

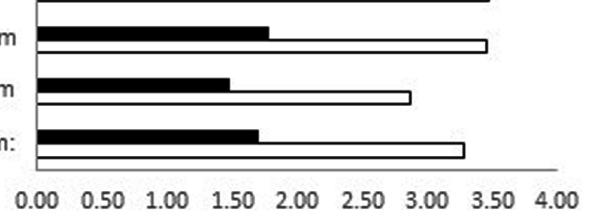

- $\mathrm{pH} 4.5$ _ $24 \mathrm{~h}$ 口pH $4.5 \_1 \mathrm{~h}$

Fig. 1. (A) Heat map showing the differentially expressed genes in Lactobacillus rhamnosus GG (LGG) exposed to acid stress at pH 4.5 for $1 \mathrm{~h}$ or $24 \mathrm{~h}$. Under acid stress condition, 128 genes were significantly upregulated and 100 genes were significantly downregulated. Results were expressed as log2 (fold change of acid stress LGG/fold change of control LGG). Red or blue blots indicated genes that were detected as differentially expressed with $p<0.05$. Differentially expressed genes: (B) upregulated and (C) downregulated genes in acid-stressed LGG were categorized based on biological process using the Cluster of Orthologous Groups of Proteins (COG) database. 
to acid stress. The expression of LGG_02240 (GroES gene) changed by 2.53 -fold in the $24 \mathrm{~h}$ acid-stressed LGG and 1.39-fold in the $1 \mathrm{~h}$ acid-stressed LGG, as compared to the control $(p<0.05)$. Furthermore, some transporter-encoding genes were induced in the acid-stressed LGG. Moreover, LGG_01064, which encodes 6-phospho- $\beta$-glucosidase and participates in the glycolysis pathway, was significantly $(p<0.05)$ upregulated in response to acid stress conditions. Interestingly, the transcriptomic study showed that LGG_02372, which encodes pilus-specific adhesion protein (spaF) was up-regulated by 3.34 -fold higher in $24 \mathrm{~h}$ acid-
stressed-LGG and 1.34-fold in $1 \mathrm{~h}$ acid-stressed LGG, as compared to control $(p<0.05)$.

Meanwhile, genes that were downregulated in response to acid stress conditions were mainly involved in the carbohydrate pyruvoyl-tetrahydropterin synthase (PTS) system, including those encoding the galactitol-, fructose-, mannitol-, and mannose-specific transporter subunit. Genes encoding amino acid and nucleotide transport systems, as well as various enzymes involved in biosynthesis, transfer, and proteolysis were also suppressed in response to acid stress, including the genes involved in L-lysine biosynthesis
A

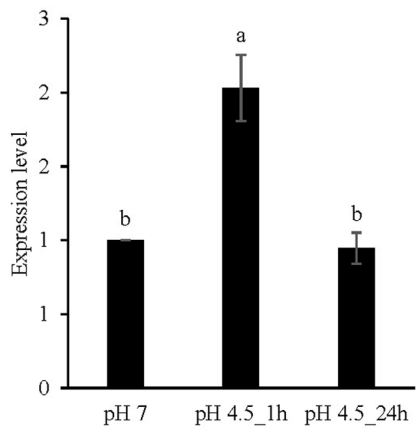

E

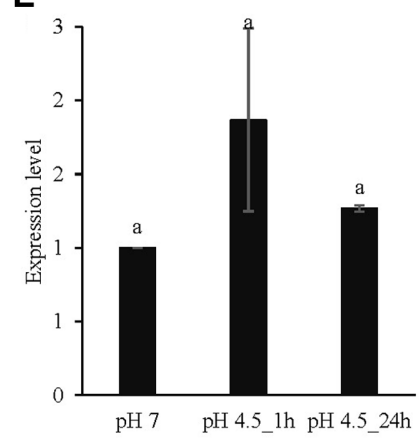

B

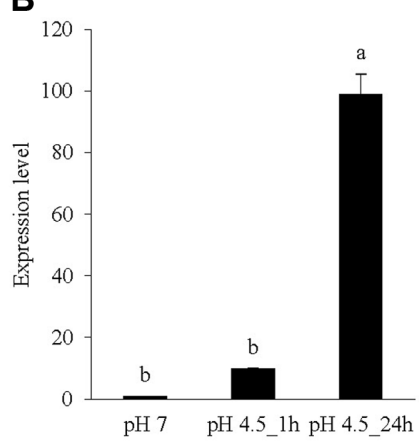

$\mathbf{F}$

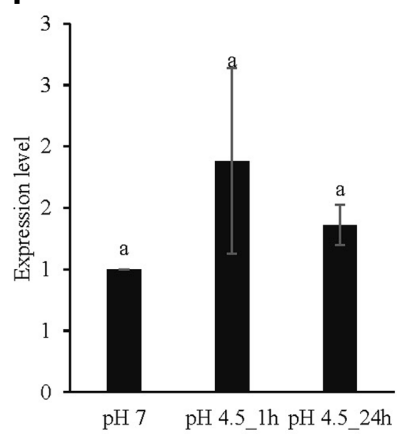

C

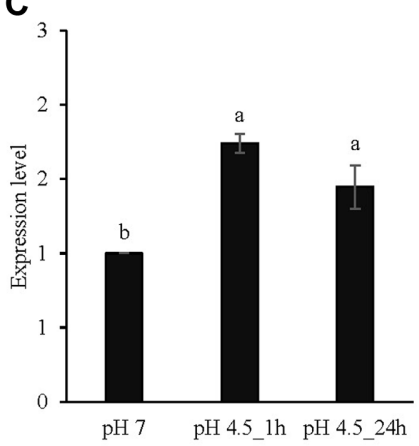

G

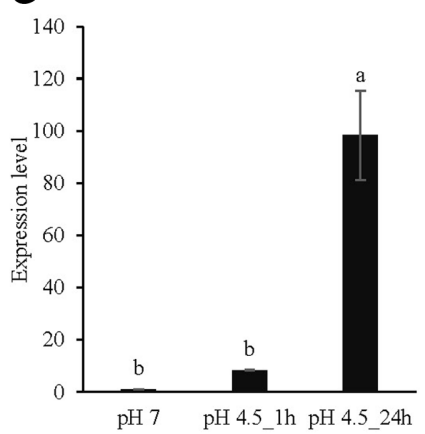

D

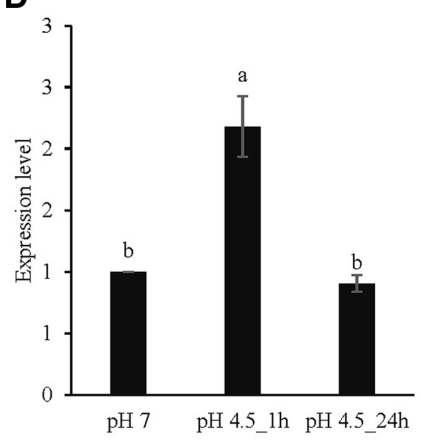

H

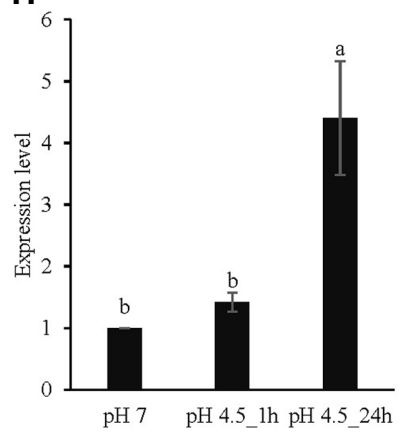

I

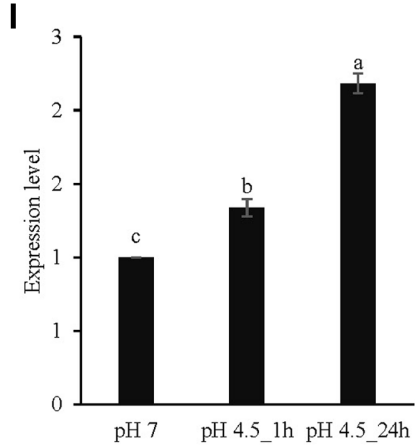

Fig. 2. Gene expression of (A) spaC, (B) spaF, (C) pili, (D) pilus444, (E) pilus443, (F) pillus442, (G) pilus2370, (H) internalin, and (I) mbf from Lactobacillus rhamnosus GG (LGG) upon exposure to acid stress at $\mathrm{pH} 4.5$ for $1 \mathrm{~h}$ or $24 \mathrm{~h}$.

The mean values of samples with different lowercase letters were significantly different $(p<0.05)$. 
via the diaminopimelate (DAP) pathway (LGG_00113, LGG_00115, LGG_00108, and LGG_00109). The transcriptomics analysis also revealed drastic differences in the profiles of downregulated genes between the different exposure times ( 1 and $24 \mathrm{~h}$ ) to acid at $\mathrm{pH} 4.5$. The expression of these genes was greatly suppressed after exposure to acid stress for $1 \mathrm{~h}$ and gradually recovered towards normal levels after exposure to acid stress for $24 \mathrm{~h}$ (Fig. 1C).

\section{The Expression Levels of Adhesion-Related Genes under Acid Stress}

The gene expression analysis revealed that the expression of all the adhesion-related genes was affected upon exposure to acid at $\mathrm{pH} 4.5$, and the exposure time played a key role in the regulation of these adhesion-related genes in LGG (Fig. 2). The gene expression of $s p a C$ (Fig. 2A), which is a component of the spaCBA pili gene cluster, was significantly upregulated (2.03 \pm 0.23 -fold) upon exposure to acid stress for $1 \mathrm{~h}$, while its expression returned towards a normal level after exposure to acid stress for $24 \mathrm{~h}$. Meanwhile, the gene expression of spaF (Fig. 2B), which is another pilus gene cluster in LGG, increased $(p<0.05)$ by $9.88 \pm 0.29$-fold after exposure to acid stress for $1 \mathrm{~h}$, and sharply increased $(p<0.05)$ by $98.99 \pm 6.60$-fold after exposure to acid stress for $24 \mathrm{~h}$. These findings indicate that the expression of spaCBA and spaFED was highly associated with the duration of exposure to acid stress. Similar to the expression of $\mathrm{spaC}$, that of pilus 444 was significantly upregulated by $2.18 \pm$ 0.25 -fold upon exposure to acid stress for $1 \mathrm{~h}$ and returned to a normal level after exposure to acid stress for $24 \mathrm{~h}$ (Fig. 2D). Meanwhile, the expression levels of pilus2370 (Fig. 2G) and internalin (Fig. 2H), which is a mucus-binding protein, only altered upon exposure to acid stress for $24 \mathrm{~h}$, showing significant increase of $98.35 \pm 17.08$-fold and 4.41 \pm 0.92 -fold, respectively. In addition, $m b f$, which encodes cell-wall anchor mucin-binding factor, was significantly upregulated by $1.34 \pm 0.06$-fold upon exposure to acid stress for $1 \mathrm{~h}$ and by $2.18 \pm 0.07$-fold upon exposure to acid stress for $24 \mathrm{~h}$ (Fig. 2I).

\section{Mucin-Binding Property of Acid-Stressed LGG}

To assess the reliability of the transcriptomic and gene expression analyses, the mucin-binding ability of LGG upon acid stress was investigated. The results showed a positive correlation between the transcriptomic and gene expression data and the mucin-binding ability of LGG. The number of LGG cells attached to the mucin layer was $1 \log \mathrm{CFU} / \mathrm{ml}$ higher $(p<0.05)$ in the acid-stressed LGG, with $6.32 \log \mathrm{CFU} / \mathrm{ml}$ in the $1 \mathrm{~h}$ acid-stressed LGG and

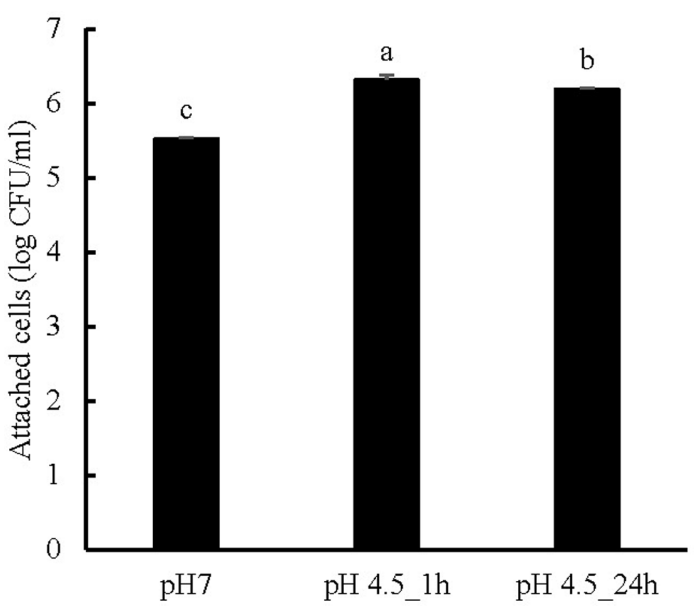

Fig. 3. The ability of acid-stressed Lactobacillus rhamnosus GG (LGG) to adhere to mucin protein.

Results are expressed as the means \pm standard deviation $(n=3)$. The means values of LGG treatments with different lowercase letters were significantly different $(p<0.05)$.

$6.19 \log \mathrm{CFU} / \mathrm{ml}$ in the $24 \mathrm{~h}$ acid-stressed LGG, as compared to $5.52 \log \mathrm{CFU} / \mathrm{ml}$ in the control (Fig. 3).

\section{In Vivo Assessment of the Intestine-Binding Ability of Acid-Stressed LGG}

The intestine-binding ability of acid-stressed LGG was determined by detecting the fluorescence expression of FITC-labeled LGG cells that were attached to mice intestines. A stronger fluorescent signal was detected in the acidstressed LGG as compared to the pH 7.0-treated LGG and the non-treated LGG control in the ileum, caecum and colon samples, indicating that the acid-stressed LGG displayed a greater ability to adhere to the intestinal wall (Fig. 4). The enhanced adherence capacity of the acidstressed LGG was further illustrated by the finding of a significantly higher number of viable LGG in the acidstressed samples as compared to pH 7.0-treated LGG and non-treated LGG control in the ileum, caecum, and colon of the mice (Fig. 5). The number of viable acid-stressed LGG cells was $1 \log \mathrm{CFU} / \mathrm{g}$ greater $(p<0.05)$ than that of the $\mathrm{pH}$ 7.0-treated LGG and $2 \log$ CFU/g greater $(p<0.05)$ than that of the non-treated LGG in ileum samples. In caecum samples, the number of viable acid-stressed LGG cells was $2 \log \mathrm{CFU} / \mathrm{g}$ higher $(p<0.05)$ than that of both the $\mathrm{pH} 7.0-$ treated and non-treated LGG. In colon samples, the number of viable acid-stressed LGG was $2 \log$ CFU/g higher $(p<$ $0.05)$ than that of the $\mathrm{pH} 7.0$-treated LGG and $3 \log \mathrm{CFU} / \mathrm{g}$ higher $(p<0.05)$ than that of the non-treated LGG. 


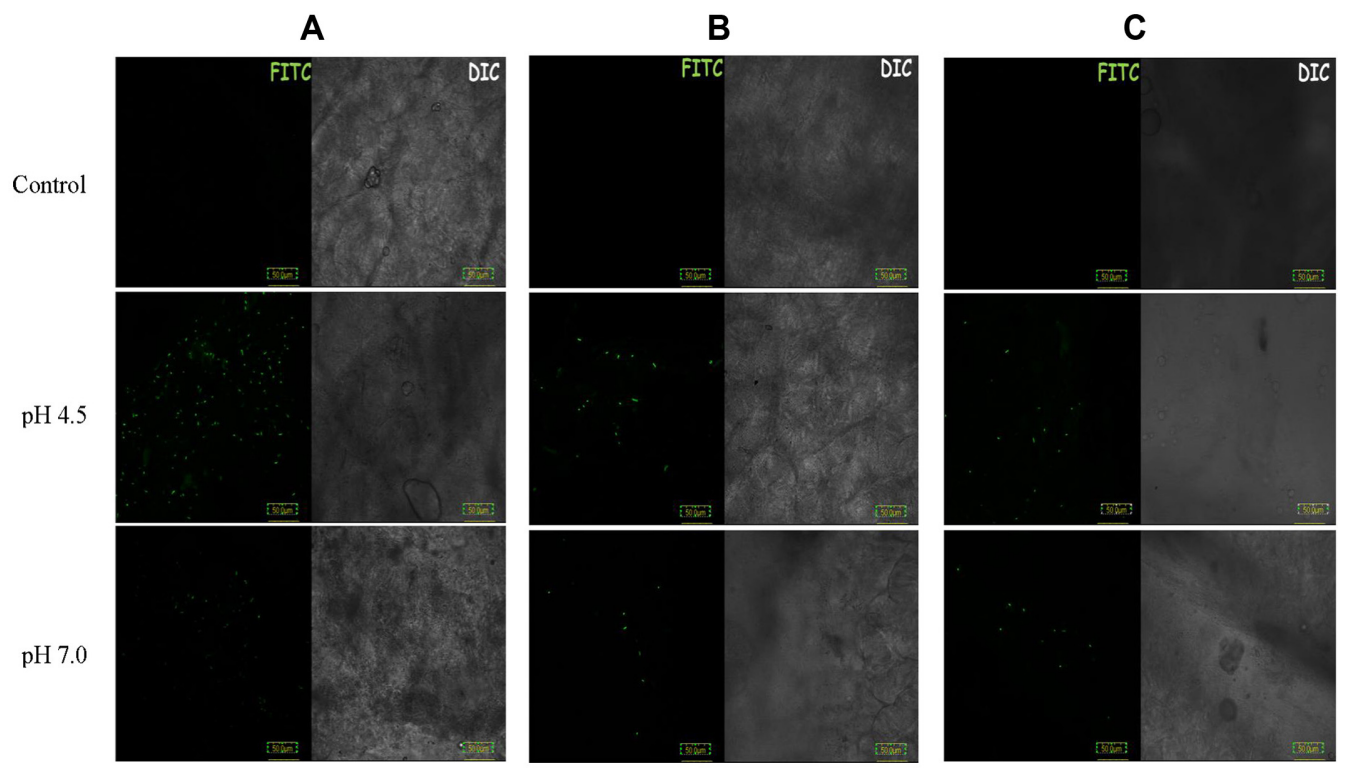

Fig. 4. Fluorescence expression of orally administered acid-stressed Lactobacillus rhamnosus GG (LGG) adhered to the (A) ileum, (B) caecum, and (C) colon of mice.

The LGG cells were labeled with fluorescein isothiocyanate (FITC) solution (1 mg/ml in $50 \mathrm{mmol} / 1$ sodium carbonate buffer, $\mathrm{pH} 8.9)$ before being administered to the mice. Control, non-treated cells; $\mathrm{pH} 4.5$, treated with $\mathrm{pH} 4.5$ medium for $24 \mathrm{~h}$; $\mathrm{pH} 7.0$, treated with $\mathrm{pH} 7.0$ medium.

\section{Discussion}

The ability of probiotic bacteria to withstand and survive the acidic environment of the mammalian gut is of paramount importance for them to reach and adhere to the intestinal walls as part of the gut microbiota. As a functional probiotic that has been extensively used in fermented foods and dietary supplements, LGG tolerates acid stress well and has several defense mechanisms to ensure its survivability. A previous study demonstrated the excellent acid tolerance ability of LGG, which had a high survival rate after 52 weeks of storage in yogurt (as a representative acidic fermented dairy products [1]. The mechanisms of acid resistance in lactic acid bacteria have been well documented, including the modulation of the proton-translocating ATPase to maintain the cytoplasmic $\mathrm{pH}$ homeostasis, the transition of pyruvate metabolism towards acetyl-CoA production, and the alteration of amino
A

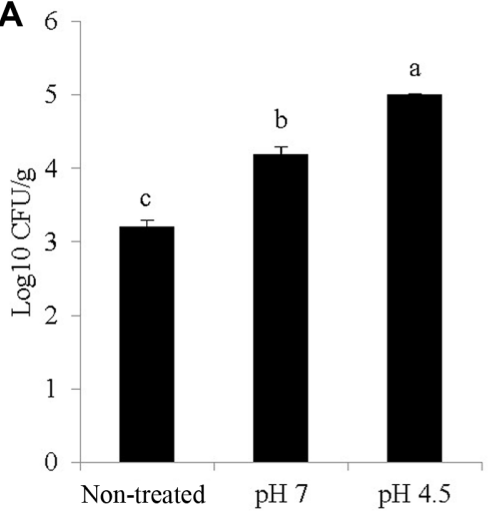

B

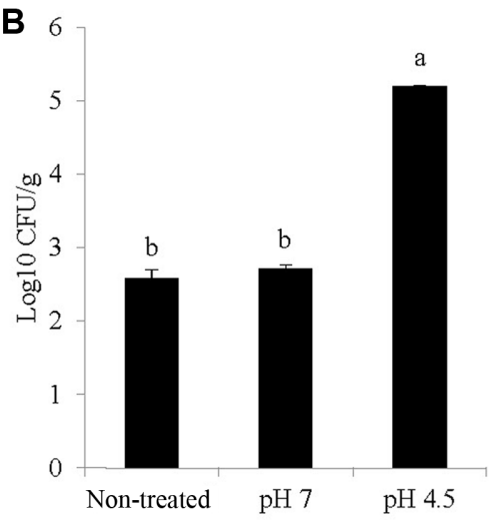

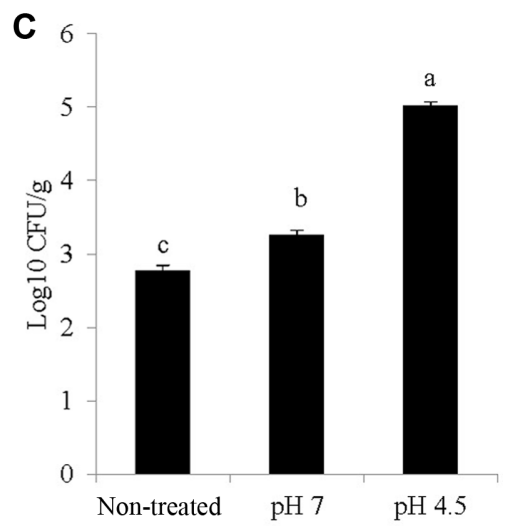

Fig. 5. The viability of the orally administered acid-stressed Lactobacillus rhamnosus GG (LGG) in the (A) ileum, (B) caecum, and (C) colon of the mice. Results are expressed as the means \pm standard deviation $(n=3)$.

The means values of LGG treatments with different lowercase letters were significantly different $(p<0.05)$. 
acid biosynthesis to supply energy for resisting acid stress $[1,8,22]$.

In this study, differences in the gene expression of LGG after exposure to acidic conditions for 1 or $24 \mathrm{~h}$ were investigated. Our transcriptomic data showed that the expression of general stress response genes was elevated in the acid-stressed LGG. Our results agree with those of other studies that have shown the importance of several stress response genes during acid stress. For instance, the activation of co-chaperonin ES ( $g r o E S$ ) is essential for acidstressed LGG to acquire tolerance and adapt to acidic environments through proper protein renaturation, folding, and denaturation $[13,23,24]$. In addition to the widely reported heat shock proteins, in the present study, the cold shock protein-encoding gene, $\operatorname{csp} \mathrm{C}$ was also found to be upregulated, as a consequence of the accumulation of denatured proteins during acid stress [24]. Notably, genes encoding phage-related proteins in acid-stressed LGG were greatly upregulated, which indicates that a mechanism for evolving and adapting to acidic environment was acquired by LGG via horizontal gene transfer [13].

In response to acid stress, the PTS carbohydrate transport genes of LGG were downregulated. Numerous studies have reported the alteration of carbohydrate metabolism under condition of acid stress, which enables bacteria to resist acid stress better by increasing their supply of energy [22, 25]. Similar findings were observed in this study, where several genes, including LGG_01064, which encodes 6phospho- $\beta$-glucosidase that hydrolyzes 6-phospho- $\beta$-Dglucosyl-(1,4)-D-glucose into D-glucose and D-glucose-6phosphate, the two substrates used in glycolysis for energy generation, were upregulated under acid stress conditions. On the contrary, genes whose products catalyze the reverse reaction of glycolysis, for instance, the fructose-1,6bisphosphatase encoding gene (LGG-02032) and the transaldolase encoding gene (LGG_00418), were apparently downregulated, to conserve energy for resisting acid stress. This conserved energy and the ATP generated from glycolysis are used to induce the $\mathrm{F}_{1} \mathrm{~F}_{0}$-ATPase proton pump and regulate the internal $\mathrm{pH}$ of LGG, as demonstrated in the previous study [1]. To maintain the anion pool within the cytoplasm, the ammonium transporter, amtB (LGG_00633), was also downregulated to preserve ammonium ions for intracellular homeostasis purposes.

The amino acid metabolism of LGG was also affected by acid stress. Amino acid metabolism has several physiological roles in lactic acid bacteria, including the control of intracellular $\mathrm{pH}$, the generation of metabolic energy or redox power, and the resistance to stress [26]. Previous studies have reported that aspartate could protect lactic acid bacteria against acid stress [26, 27]. Our transcriptomic analysis of the acid-stressed LGG demonstrated decreases in the gene expression of aspartate kinase (lys; LGG_00113), aspartate semialdehyde dehydrogenase (asd; LGG_00115), dihydrodipicolinate synthase (dapA; LGG_00109) and dihydrodipicolinate reductase (dapB; LGG_00108), indicating that the biosynthesis of L-lysine from aspartate via DAP pathway was suppressed. Fernandez and Zuniga [26] suggested that aspartate can be converted to arginine to increase the intracellular $\mathrm{pH}$ of $L$. casei. Wu et al. (2013) demonstrated that aspartate supplementation increased the production of $\mathrm{NH}_{4}^{+}$, which subsequently neutralized intracellular protons and raised the cytosolic $\mathrm{pH}$, resulting in an enhanced resistance to acid stress in L.casei [27]. Hence, the conservation of aspartate via the suppression of L-lysine biosynthesis was suggested to be associated with the improved acid stress resistance in LGG.

Another important finding from the transcriptomic analysis of acid-stressed LGG was the upregulation of spaF gene (LGG_02372), which encodes the pilus-specific adhesion protein. It is crucial for LGG to adhere and maintain persistent growth in the gastrointestinal tract so that it can displace pathogens, and exert immunomodulatory effects on the host. Since spaF gene encodes the main adhesion determining SpaF pilin of the spaFED pilus cluster, its presence and activation mediate and enhance the binding of cells towards the intestinal tract [18, 28, 29]. Although LGG possesses two pilus gene clusters, spaCBA and spaFED, only the SpaCBA pili cluster is commonly detected under laboratory conditions $[17,29]$. It was suggested that the absence of the SpaFED pili cluster under laboratory conditions is likely due to a lack of appropriate environmental stimuli to activate the expression of the spaFED genes, similar to the Tad pilus genes in Bifidobacterium breve that are only expressed in mouse intestine [17, 30].

The outcomes of the transcriptomic analysis of acidstressed LGG were further confirmed via gene expression analysis. The common SpaCBA pili cluster gene, spaC was only upregulated after acid stress treatment for $1 \mathrm{~h}$. Meanwhile, the expression of spaF gene, which encodes the SpaFED pili cluster, increased by 10 -fold upon acid stress treatment for $24 \mathrm{~h}$, indicating that prolonged exposure to acid stress at $\mathrm{pH} 4.5$ could enhance the expression of mucus-binding pili in LGG. In addition, the genes encoding mucus-binding factor, $m b f$ gene, and the internalin were also upregulated upon exposure to acid stress for $24 \mathrm{~h}$. The presence and expression of these pili-related genes contribute to the adhesion and residency time of LGG in the human 
gastrointestinal tract, since a previous study reported that the residency time of pilus-deficient $L$. rhamnosus was considerably shorter than that of wild-type L. rhamnosus in a human intervention study [18]. Based on our results, we postulated that the exposure of LGG cells to acid stress enhances their adhesion by stimulating the expression of spaC and spaF genes for mucosal adhesion, and by inducing mucus-binding factor ( $m b f$ gene) and internalin gene, which substantially augment the competitiveness of LGG cells for the ecological niches of the human gastrointestinal tract.

Following the transcriptomic and gene expression analyses, the mucin and gastrointestinal tract-binding abilities of LGG under acid stress conditions were further justified via in vitro and in vivo analyses. A previous study showed that wild-type LGG displayed high adhesion capacities towards intestinal epithelial cells and mucin [31]. Considering that LGG is currently the only known probiotic strain with mucus-binding pili, an enhancement of the activities of the SpaCBA and SpaFED mucus-binding pili could further improve the competitiveness of LGG in the mucosal environment as compared to other probiotic strains [17]. The previous study also reported the importance of pili in facilitating the adherence ability of LGG to human intestinal tissue and thereby prolonging the retention of LGG during their transit through the gastrointestinal tract. This is important because only adherent probiotics can effectively exert immunomodulatory effects and stabilize the intestinal mucosal barrier $[14,18]$. In this study, we found that the acid-stressed LGG exhibited a higher capacity for adhesion to mucin. Owing to the inability of in vitro assays to resemble the complex environmental conditions of cells within a living organism, an animal study was conducted to evaluate the effect of acid stress on the adherence and retention of LGG during transit through the gastrointestinal tract. The results of the in vivo study confirmed the enhanced adhesion of LGG in the gastrointestinal tract under acid stress conditions, indicating that acid stress could enhance the adhesion of LGG and thereby prolong its retention in the gastrointestinal tract, enabling it to exert its health-promoting properties.

The findings presented in this study provide a detailed understanding of the adaptation of LGG towards acid stress conditions, including the enhancement of its adhesion ability (Fig. 6). Our findings lead us to a novel discovery regarding the enhanced adhesion properties of LGG upon acid stress treatment, especially the expression of the spaFED pili gene cluster, which was previously undetectable in LGG cultured outside the mammalian gastrointestinal tract. Based on our results, exposure to acid stress conditions

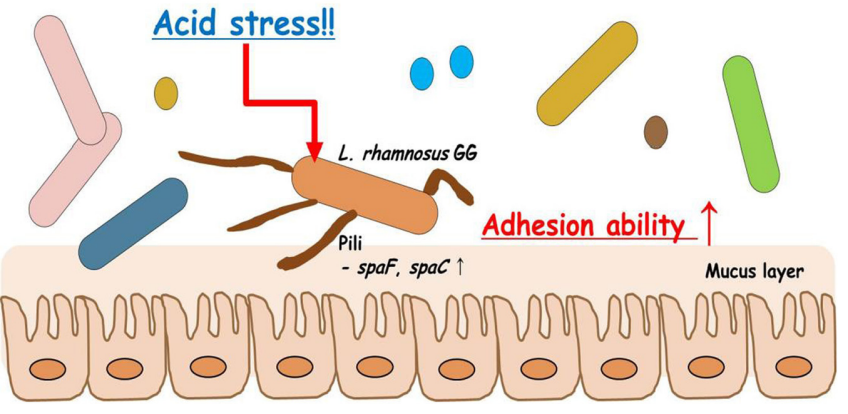

Fig. 6. Interaction of Lactobacillus rhamnosus GG (LGG) subjected to acid stress conditions with epithelial cells in the mucosa.

Upon exposure to an acidic environment, pili genes of LGG such as $s p a F$ and $s p a C$ were highly expressed and the cells bound strongly to the gastrointestinal mucus layer of the host. These results indicated that the adhesion ability of LGG was enhanced after exposure to acid stress conditions.

similar to those in fermented dairy products enhanced the adhesion properties of LGG, to confer a competitive advantage in the mucosal environment for which to outcompete pathogens with advanced probiotic properties.

\section{Acknowledgments}

This work was supported by the Basic Science Research Program through the National Research Foundation of Korea (NRF) funded by the Ministry of Education, Science, and Technology (NRF-2016R1A2B4007519).

\section{Conflict of Interest}

The authors have no financial conflicts of interest to declare.

\section{References}

1. Bang M, Oh S, Lim KS, Kim Y, Oh S. 2014. The involvement of ATPase activity in the acid tolerance of Lactobacillus rhamnosus strain GG. Int. J. Dairy Technol. 67: 229-236.

2. Lambert R, Stratford M. 1999. Weak-acid preservatives: modelling microbial inhibition and response. J. Appl. Microbiol. 86: $157-164$.

3. Serrazanetti DI, Guerzoni ME, Corsetti A, Vogel R. 2009. Metabolic impact and potential exploitation of the stress reactions in lactobacilli. Food Microbiol. 26: 700-711.

4. Choi SH, Baumler DJ, Kaspar CW. 2000. Contribution of dps to acid stress tolerance and oxidative stress tolerance in Escherichia coli O157: H7. Appl. Environ. Microbiol. 66: 3911- 
3916.

5. Warnecke T, Gill RT. 2005. Organic acid toxicity, tolerance, and production in Escherichia coli biorefining applications. Microb. Cell Fact. 4: 25.

6. Duary RK, Batish VK, Grover S. 2010. Expression of the atpD gene in probiotic Lactobacillus plantarum strains under in vitro acidic conditions using RT-qPCR. Res. Microbiol. 161: 399-405.

7. Kullen MJ, Klaenhammer TR. 1999. Identification of the $\mathrm{pH}-$ inducible, proton-translocating F1F0-ATPase (atpBEFHAGDC) operon of Lactobacillus acidophilus by differential display: gene structure, cloning and characterization. Mol. Microbiol. 33: $1152-1161$.

8. Cotter PD, Hill C. 2003. Surviving the acid test: responses of gram-positive bacteria to low pH. Microbiol. Mol. Biol. Rev. 67: 429-453.

9. Broadbent JR, Larsen RL, Deibel V, Steele JL. 2010. Physiological and transcriptional response of Lactobacillus casei ATCC 334 to acid stress. J. Bacteriol. 192: 2445-2458.

10. Graham JW, Lei MG, Lee CY. 2013. Trapping and identification of cellular substrates of the Staphylococcus aureus ClpC chaperone. J. Bacteriol. 195: 4506-4516.

11. Varmanen P, Vogensen FK, Hammer K, Palva A, Ingmer H. 2003. ClpE from Lactococcus lactis promotes repression of CtsR-dependent gene expression. J. Bacteriol. 185: 5117-5124.

12. Suokko A, Poutanen M, Savijoki K, Kalkkinen N, Varmanen P. 2008. ClpL is essential for induction of thermotolerance and is potentially part of the $H r c A$ regulon in Lactobacillus gasseri. Proteomics 8: 1029-1041.

13. Wall T, Båth K, Britton RA, Jonsson H, Versalovic J, Roos S. 2007. The early response to acid shock in Lactobacillus reuteri involves the ClpL chaperone and a putative cell wallaltering esterase. Appl. Environ. Microbiol. 73: 3924-3935.

14. Salminen S, Isolauri E, Salminen E. 1996. Probiotics and stabilisation of the gut mucosal barrier. Asia Pac. J. Clin. Nutr. 5: 53-56.

15. Di Caro S, Tao H, Grillo A, Elia C, Gasbarrini G, Sepulveda A, et al. 2005. Effects of Lactobacillus GG on genes expression pattern in small bowel mucosa. Dig. Liver Dis. 37: 320-329.

16. Oksaharju A, Kankainen M, Kekkonen RA, Lindstedt KA, Kovanen PT, Korpela R, et al. 2011. Probiotic Lactobacillus rhamnosus downregulates FCER1 and HRH4 expression in human mast cells. World J. Gastroenterol. 17: 750-759.

17. Reunanen J, von Ossowski I, Hendrickx AP, Palva A, de Vos WM. 2012. Characterization of the SpaCBA pilus fibers in the probiotic Lactobacillus rhamnosus GG. Appl. Environ. Microbiol. 78: 2337-2344.

18. Kankainen M, Paulin L, Tynkkynen S, von Ossowski I, Reunanen J, Partanen P, et al. 2009. Comparative genomic analysis of Lactobacillus rhamnosus GG reveals pili containing a human-mucus binding protein. Proc. Natl. Acad. Sci. USA 106: $17193-17198$.

19. Mandlik A, Swierczynski A, Das A, Ton-That H. 2008. Pili in Gram-positive bacteria: assembly, involvement in colonization and biofilm development. Trends Microbiol. 16: 33-40.

20. Proft T, Baker E. 2009. Pili in Gram-negative and Grampositive bacteria-structure, assembly and their role in disease. Cell Mol. Life Sci. 66: 613-635.

21. Tynkkynen S, Singh KV, Varmanen P. 1998. Vancomycin resistance factor of Lactobacillus rhamnosus GG in relation to enterococcal vancomycin resistance (van) genes. Int. J. Food Microbiol. 41: 195-204.

22. Wu R, Zhang W, Sun T, Wu J, Yue X, Meng H, et al. 2011. Proteomic analysis of responses of a new probiotic bacterium Lactobacillus casei Zhang to low acid stress. Int. J. Food Microbiol. 147: 181-187.

23. Rallu F, Gruss A, Maguin E. 1996. Lactococcus lactis and stress. Antonie van Leeuwenhoek 70: 243-251.

24. Lim EM, Ehrlich SD, Maguin E. 2000. Identification of stress-inducible proteins in Lactobacillus delbrueckii subsp. bulgaricus. Electrophoresis 21: 2557-2561.

25. Koponen J, Laakso K, Koskenniemi K, Kankainen M, Savijoki K, Nyman TA, et al. 2012. Effect of acid stress on protein expression and phosphorylation in Lactobacillus rhamnosus GG. J. Proteom. 75: 1357-1374.

26. Fernández M, Zúñiga M. 2006. Amino acid catabolic pathways of lactic acid bacteria. Crit. Rev. Microbiol. 32: 155-183.

27. Wu C, Zhang J, Du G, Chen J. 2013. Aspartate protects Lactobacillus casei against acid stress. Appl. Microbiol. Biotechnol. 97: 4083-4093.

28. Rintahaka J, Yu X, Kant R, Palva A, von Ossowski I. 2014. Phenotypical analysis of the Lactobacillus rhamnosus GG fimbrial spaFED operon: surface expression and functional characterization of recombinant SpaFED pili in Lactococcus lactis. PLoS One 9: e113922.

29. von Ossowski I, Reunanen J, Satokari R, Vesterlund S, Kankainen M, Huhtinen $\mathrm{H}$, et al. 2010. Mucosal adhesion properties of the probiotic Lactobacillus rhamnosus GG SpaCBA and SpaFED pilin subunits. Appl. Environ. Microbiol. 76: 2049-2057.

30. Motherway MOC, Zomer A, Leahy SC, Reunanen J, Bottacini F, Claesson MJ, et al. 2011. Functional genome analysis of Bifidobacterium breve UCC2003 reveals type IVb tight adherence (Tad) pili as an essential and conserved host-colonization factor. Proc. Natl. Acad. Sci. USA 108: 11217-11222.

31. Doron S, Snydman DR, Gorbach SL. 2005. Lactobacillus GG: bacteriology and clinical applications. Gastroenterol. Clin. North Am. 34: 483-498. 